\title{
Correction to: Impact of Oral Abrocitinib Monotherapy on Patient-Reported Symptoms and Quality of Life in Adolescents and Adults with Moderate-to-Severe Atopic Dermatitis: A Pooled Analysis of Patient-Reported Outcomes
}

\author{
Jonathan I. Silverberg ${ }^{1} \cdot$ Jacob P. Thyssen $^{2} \cdot$ Eric L. Simpson $^{3} \cdot$ Gil Yosipovitch $^{4} \cdot$ Sonja Ständer $^{5} \cdot$ Hernan Valdez $^{6}$. \\ Ricardo Rojo $^{7}$. Pinaki Biswas ${ }^{6}$. Daniela E. Myers ${ }^{8} \cdot$ Claire Feeney $^{9} \cdot$ Marco DiBonaventura $^{6}$ (1)
}

Published online: 18 August 2021

(c) Springer Nature Switzerland AG 2021

\section{Correction to: \\ American Journal of Clinical Dermatology (2021) 22: \\ 541-554 \\ https://doi.org/10.1007/s40257-021-00604-9}

Page 550, Fig. 4:

The legend, which previously read:

Fig. 4. Impact of treatment on a Dermatology Life Quality Index (DLQI) ${ }^{\mathrm{a}}$ and $\mathbf{b}$ Children's Dermatology Life Quality Index (CDLQI) ${ }^{\mathrm{b}}$ band descriptors. ${ }^{\mathrm{a}}$ For patients $\geq 8$ years of age. ${ }^{b}$ For patients $<18$ years of age.

Should read:

The original article can be found online at https://doi.org/10.1007/ s40257-021-00604-9.

Marco DiBonaventura

marco.dibonaventura@pfizer.com

1 The George Washington University School of Medicine and Health Sciences, Washington, DC, USA

2 Bispebjerg Hospital, University of Copenhagen, Copenhagen, Denmark

3 Oregon Health and Science University, Portland, OR, USA

4 Miami Itch Center, Miller School of Medicine, University of Miami, Miami, FL, USA

5 Center for Chronic Pruritus, University of Münster, Münster, Germany

6 Pfizer Inc., New York, NY, USA

7 Pfizer Inc., Groton, CT, USA

8 Pfizer Inc., Collegeville, PA, USA

9 Pfizer Ltd., Surrey, UK
Fig. 4. Impact of treatment on a Dermatology Life Quality Index (DLQI) ${ }^{\mathrm{a}}$ and $\mathbf{b}$ Children's Dermatology Life Quality Index (CDLQI) ${ }^{\mathrm{b}}$ band descriptors. ${ }^{\mathrm{a}}$ For patients $\geq 18$ years of age. ${ }^{b}$ For patients $<18$ years of age. 